\title{
Bleaching Agent Action on Color Stability, Surface Roughness and Microhardness of Composites Submitted to Accelerated Artificial Aging
}

\author{
Raphael Mendes Bezerra Rattacaso ${ }^{a}$ \\ Lucas da Fonseca Roberti Garciab \\ Fabiano Gamero Aguilar ${ }^{c}$ \\ Simonides Consani ${ }^{\mathrm{d}}$ \\ Fernanda de Carvalho Panzeri Pires-de-Souza ${ }^{e}$
}

\section{ABSTRACT}

Objectives: The purpose of this study was to evaluate the bleaching agent action on color stability, surface roughness and microhardness of composites (Charisma, Filtek Supreme and Heliomolar A2) submitted to accelerated artificial aging (AAA).

Methods: A Teflon matrix $(12 \times 2 \mathrm{~mm})$ was used to fabricate 18 specimens $(\mathrm{n}=6)$ which, after polishing (Sof-Lex), were submitted to initial color reading $(\Delta E)$, Knoop microhardness (KHN) ( $50 \mathrm{~g} / 15$ $s$ load) and roughness $\left(R_{a}\right.$ ) (cut-off $0.25 \mathrm{~mm}$ ) tests. Afterwards, the samples were submitted to AAA for 384 hours and new color, microhardness and roughness readings were performed. After this, the samples were submitted to daily application (4 weeks) of 16\% Carbamide Peroxide (NiteWhite ACP) for 8 hours and kept in artificial saliva for 16 hours. New color, microhardness and roughness readings were made at the end of the cycle, and 15 days after bleaching.

Results: Comparison of the $\triangle \mathrm{E}$ means (2-way ANOVA, Bonferroni, $\mathrm{P}<.05$ ) indicated clinically unacceptable color alteration for all composites after AAA, but without significant difference. Statistically significant increase in the KHN values after AAA was observed, but without significant alterations 15 days after bleaching. For $\mathrm{R}_{\mathrm{a}}$ there was no statistically significant difference after AAA and 15 days after bleaching.

Conclusions: The alterations promoted by the bleaching agent and AAA are material dependent. (Eur J Dent 2011;5:143-149)

Key words: Bleaching agent; Accelerated aging; Color stability; Microhardness; Surface roughness.

- a Graduate Student, Department of Dental Materials and Prosthodontics, Dental School of Ribeirão Preto, University of São Paulo, Ribeirão Preto, SP, Brazil.

b Post-Graduate Student, Department of Restorative Dentistry, Dental Materials Area, Dental School of Piracicaba, State University of Campinas, Piracicaba, SP, Brazil.

Post-Graduate Student, Department of Dental Materials and Prosthodontics, Dental School of Ribeirão Preto, University of São Paulo, Ribeirão Preto, SP, Brazil.

d Full Professor, Department of Restorative Dentistry, Dental Materials Area, Dental School of Piracicaba, State University of Campinas, Piracicaba, SP, Brazil.
- Associate Professor, Department of Dental Materials and Prosthodontics, Dental School of Ribeirão Preto, University of São Paulo, Ribeirão Preto, SP, Brazil.

- Corresponding author:

Profa. Dra. Fernanda de Carvalho Panzeri

Pires-de-Souza,

Faculdade de Odontologia de Ribeirão Preto/USP, Departamento de Materiais Dentários e Prótese, Avenida do Café, s/n, Bairro Monte Alegre, 14040-904 Ribeirão Preto, SP, Brazil.

Phone: +55-16-3602-3973

Fax: +55-016-3633-0999

E-mail: ferpanzeriđforp.usp.br 


\section{INTRODUCTION}

Color alteration of composites can be caused by intrinsic or extrinsic factors. Intrinsic factors are related to physical chemical reactions in the deepest portions of the restoration, in addition to changes in temperature and humidity, and extrinsic factors are related to adsorption or absorption of staining substances. ${ }^{1}$

Bleaching agents act efficiently in removing intrinsic stains from the tooth and can be used under professional supervision in a dental office (professional bleaching); or without supervision (home bleaching), performed by the patient. This procedure involves the use of carbamide peroxide solutions at concentrations between $10 \%$ and $16 \%$, and generally provides satisfactory results after a period of 2 to 6 weeks. ${ }^{2}$ These solutions are very unstable, which, in contact with saliva, immediately dissociate into $3 \%$ to $5 \%$ hydrogen peroxide and $7 \%$ to $19 \%$ urea. After this, the hydrogen peroxide is degraded into oxygen and water, while urea is degraded into ammonia and carbon dioxide. ${ }^{3-5}$ The hydroxyl radicals formed combine with the intrinsic and extrinsic stains present in the teeth and remove them through an oxidation reaction.,

Frequently, teeth restored with esthetic materials are submitted to bleaching, which may cause an alteration in the restorative material color, so that it no longer mimics the color of the tooth, making it one of the most frequent reasons for replacing restorations after bleaching. ${ }^{7}$

Several studies have tested the effect of bleaching agents on the properties of dental materials. As regards to microhardness, studies have reported an increase, ${ }^{8}$ reduction ${ }^{9}$ or even no alteration $^{10-14}$ in the surface hardness of composites after the carbamide peroxide gel application.

Regarding the surface texture of the restorative material, some studies ${ }^{9,15}$ have observed small alterations in the surface of composites after daily immersion in bleaching gel, without statistically significant results among control and bleached groups. Nevertheless, Wattanapayungkul et al ${ }^{16}$ demonstrated the presence of several gaps in the restorative material surface after home bleaching, which may favor esthetic material staining after bleaching. ${ }^{7}$

Although bleaching agents are frequently used, there isn't a consensus about the effects of these products on restorative materials, particu- larly those that have already been in function for some time. ${ }^{10-16}$ Therefore, the aim of this study was to evaluate the effect of home bleaching agent on the color stability, surface roughness and microhardness of direct composites submitted to the Accelerated Artificial Aging procedure (AAA). Two hypotheses were tested in this study: the bleaching agent would be effective regarding bleaching the aged specimens, and its use would not alter the surface roughness and microhardness of the composites.

\section{MATERIALS AND METHODS}

Specimen preparation

Three composite resins with different filler size were used in the current study (Table 1). A Teflon matrix (12 × $2 \mathrm{~mm})$ was used to fabricate 18 specimens $(n=6)$ according to the incremental technique. Light activation was performed with a light emitting diode device (Flash Lite 1401, Discus Dental, Culver City, Ca, USA, 465/475 nm, 1400 $\mathrm{mW} / \mathrm{cm}^{2}$ ) for 40 seconds, in accordance with the manufacturer's recommendations.

Once removed from the matrix, the specimens were polished with abrasive paper disks in a descending order of granulation (Sof-Lex, 3M do Brasil, Sumaré, SP, Brazil). Next, the specimen's thickness was checked with a digital pachymeter (Digimess, São Paulo, SP, Brazil). After this, color, surface roughness and initial microhardness readouts were performed.

\section{Assessment procedures}

To color analysis, a Spectrophotometer (PCB 6807 BYK GARDNER, Geretsried, Germanyl was used. The standard observation simulated by the Spectrophotometric colorimeter was in accordance with the CIE L*a*b* system, described by Pires-de-Souza et al, ${ }^{17}$ and the readings were performed against a white standard background (White Standard Sphere for 450, $0^{\circ}$ Reflectance and Color Gardner Laboratory Inc. Bethesda, Geretsried, Germanyl. Knoop microhardness of the specimens (KHN) was determined using a microhardness tester (HMV 2000, Shimadzu, Kyoto, Japan) in three different points, with a $50 \mathrm{~g} / 15 \mathrm{~s}$ load. Surface roughness $\left(R_{a}\right)$ was measured with a Rugosimeter Surfcorder SE 1700, cut-off - 0.25 mm (Kosakalab, Tokyo, Japan). The rugosimeter needle was positioned over each test specimen, 
performing three readings in different locations of the sample surface. After the three readings, the mean surface roughness values were obtained.

\section{Aging procedure}

After initial color, surface roughness and microhardness analyses, the specimens were submitted to Accelerated Artificial Aging (AAA) using an Accelerated Aging System for non-metallic objects C-UV (Comexim Matérias Primas Ltda., São Paulo, Brazil), under the action of UV light and condensation, which were activated in separate cycles repeated successively and automatically. The AAA is achieved in a laboratory environment that indicates the behavior of materials under certain conditions and it is widely used for development and control of different materials properties. ${ }^{18}$ This system is composed of a network of eight fluorescent light tubes of 40 watts with emission concentrated in the ultraviolet $B$ region; with radiation concentrated at $280 / 32 \mathrm{~nm}$ and the exposure temperature is automatically controlled according to the programs established by the UV/ condensation cycles. The working program was standardized for 4 hours of exposure to UV-B at $50^{\circ} \mathrm{C}$ and 4 hours of condensation at $50^{\circ} \mathrm{C}$. The distance from the light source was $50 \mathrm{~mm}$ and the maximum aging time 384 hours ${ }^{17}$ corresponding to a year of clinical use, ${ }^{19}$ in agreement with the guidelines recommended by ASTM. ${ }^{18}$

After AAA, new color, surface roughness and microhardness readings were performed, following the same methodology. After this, the specimens were submitted to daily application
(4 weeks) of $16 \%$ Carbamide Peroxide (NiteWhite ACP Discus Dental, Culver City, CA, USA, pH 5.9 to 6.6) for 8 hours in oven at $37^{\circ} \mathrm{C}$. After this period, the specimens were washed under running water and kept in artificial saliva at $37^{\circ} \mathrm{C}$ for 16 hours. At the end of the cycle, new color, microhardness and roughness readings were performed, and again, 15 days after bleaching. In this period, the specimens were kept in artificial saliva at $37^{\circ} \mathrm{C}$, changed every day.

The color stability $(\Delta E)$ of the materials was calculated using the formula: $\Delta \mathrm{E}^{*}=\left[\left(\Delta \mathrm{L}^{*}\right)^{2}+\left(\Delta \mathrm{a}^{*}\right)\right.$ $\left.{ }^{2}+\left(\Delta b^{*}\right)^{2}\right]^{1 / 2}$ where $\Delta E$ represents the color change in all dimensions ( $\left.\mathrm{L}^{*} \mathrm{a}^{*} \mathrm{~b}^{*}\right)$ and $\Delta \mathrm{L}^{*}, \Delta \mathrm{a}^{*} \Delta \mathrm{b}^{*}$ represent color changes along the individual axes. ${ }^{20}$ Values of $\Delta E \geq 3.3$ were considered clinically unacceptable. ${ }^{21}$

The initial and after-treatment values of $\Delta E$, surface roughness and microhardness were statistically analyzed (2-way ANOVA, Bonferroni's test, $P<.05)$.

\section{RESULTS}

\section{Color spectrophotometry}

The comparisons between the intervals of aging and baseline, bleaching and baseline; and 15 days before bleaching and baseline are shown in Table 2. It was verified that Charisma and Heliomolar presented high $\triangle \mathrm{E}$ values after $\mathrm{AAA}$, an alteration considered clinically unacceptable, ${ }^{21}$ with statistically similar results between them ( $P>$.05). After AAA, the composite Filtek Supreme presented color alteration with $\Delta \mathrm{E}$ considered within the acceptable limit, ${ }^{21}$ with statistically significant

Table 1. Tested composites.

\begin{tabular}{lcccc}
\hline Composite & Monomers & Loads & & Manufacturer \\
& & Size & \% by volume \\
\hline Charisma & Bis-GMA, TEGDMA & $2-0.04 \mu \mathrm{m}$ & Heraus Kulzer, Hanau, Germany \\
Filltek Supreme Z350 & Bis-GMA, Bis-EMA, UDMA, & $0.6-1.4 \mu \mathrm{m}$ (Agglomerated), & $59.5 \%$ & $3 M$ ESPE, Irvine, CA, USA \\
Heliomolar & TEGDMA & $5-20 \mathrm{~nm}$ (Nanoagglomerated) & Ivoclar/Vivadent AG, Schaan, \\
& Bis-GMA, UDMA & $0.04-0.2 \mu \mathrm{m}$ & $46 \%$ & Liechtenstein \\
\hline
\end{tabular}

Bis-GMA Bisphenol glycidyl methacrylate; TEGDMA: triethyleneglycol dimethacrylate; UDMA: Urethane Dimethacrylate; Bis-EMA: Bisphenol A diethoxy methacrylate.

Table 2. Means (standard deviation) of $\Delta \mathrm{E}$ of aged samples (2-way ANOVA, Bonferroni, $\mathrm{P}<.05$ )

\begin{tabular}{lccc}
\hline & Post-aging & Post-bleaching & 15 days after bleaching \\
\hline Charisma & $5.83(1.7)^{\mathrm{a}, \mathrm{A}}$ & $3.91^{(0.8)^{\mathrm{b}, \mathrm{A}}}$ & $3.79(0.9)^{\mathrm{b}, \mathrm{A}}$ \\
Filltek Supreme Z350 & $2.04(0.5)^{\mathrm{a}, \mathrm{B}}$ & $5.17(0.8)^{\mathrm{a}, \mathrm{A}}$ & $3.54(0.8)^{\mathrm{a}, \mathrm{A}}$ \\
Heliomolar & $4.48(3.1)^{\mathrm{a}, \mathrm{A}}$ & $5.17(0.8)^{\mathrm{a}, \mathrm{A}}$ & $5.18(0.8)^{\mathrm{a}, \mathrm{A}}$ \\
\hline
\end{tabular}

*Different letters, lower case letters on the line and capital letters in the column indicate statistically significant difference $(P<.05)$. 
difference in comparison with the other materials $(P<.05)$. After bleaching, Charisma presented lower $\Delta \mathrm{E}$ than after aging, a statistically significant result $(P<.05)$. For the other materials, there was an increase in $\Delta E$, without statistically significant difference $(P>$.05). When comparing the performance of composites after bleaching, there were no statistically significant differences among them $(P>$.05). Fifteen days after bleaching, the $\Delta \mathrm{E}$ values were very similar in comparison with the postbleaching values, without statistically significant differences among the materials, in comparison with the previous treatment $(P>.05)$.

\section{Surface roughness}

The results after AAA (Table 3 ) indicated that there was no statistically significant difference ( $P>$.05) in roughness for any of the tested materials. When the materials were compared among them, there was statistically significant difference $(P<.05)$ only for Heliomolar. After bleaching, Filtek Supreme and Heliomolar presented an increase in surface roughness values, with statistically significant difference $(P>.05)$ in comparison to the post AAA values. Fifteen days after bleaching, there was not statistically significant difference (P>.05) in comparison with the post bleaching situation.

\section{Microhardness}

As regards the microhardness values (Table 4), a large increase was verified after AAA, results differing statistically from the initial values $(P<.05)$. After bleaching, there was an increase in the microhardness of Heliomolar $(P<.05)$. For the other composites, there was a decrease, with statistically significant results ( $P>.05)$. After 15 days, there were no significant alterations (P>.05).

\section{DISCUSSION}

There is a consensus among researchers that direct composite resin restorations undergo color alteration with the passage of time, ${ }^{21-28}$ and that one of the greatest challenges in modern dentistry is to find a material that has a color stability similar to that of the dental structure; and that this stability can be maintained in the oral environment as the years pass. ${ }^{29-30}$

As regards the color stability, this study tested the hypothesis that the bleaching agent would promote bleaching of the studied composites. The results demonstrated that the color of composites could be significantly altered by $A A A^{31}$ and bleaching, and this alteration is material dependent, results that are similar to other studies.,32 This susceptibility to color alteration can be attributed to the composition of the resin matrix and the type and volume of load particles of the composite. ${ }^{7}$

When bleached, the aged specimens did not presented significant alteration for $\Delta E(P>.05)$, with the exception of the composite Charisma, and the same behavior was maintained 15 days after bleaching. This fact demonstrated that the action of the bleaching agent was more effective on the aged specimens, allowing one to agree with the tested hypothesis. After being bleached, the specimens remained without significant color alteration ( $P>$.05). This may have occurred due to the stability of bleaching agents and their prolonged action. $^{31}$

Differences in the chemical structure of composites, such as the type of oligomers or monomers used; concentration/type of activators, initiators, inhibitors; oxidation of carbon doublebonds; size/type of load particles and the load

Table 3. Means (standard deviation) of surface roughness (Ra) of aged samples (2-way ANOVA, Bonferroni, $\mathrm{P}<.05$ ).

\begin{tabular}{|c|c|c|c|c|}
\hline & Initial & Post-aging & Post-bleaching & 15 days after bleaching \\
\hline Charisma & $0.18(0.1)^{a, A}$ & $0.32(0.1)$ ab,AB & $0.35(0.1)^{\mathrm{b}, \mathrm{A}}$ & $0.41(0.2)^{b, A}$ \\
\hline Filltek Supreme Z350 & $0.12(0.1)^{a, A}$ & $0.12(0.0)^{a, A}$ & $0.53(0.1)^{b, A}$ & $0.51(0.1)^{b, A}$ \\
\hline Heliomolar & $0.27(0.2)^{\mathrm{a}, \mathrm{A}}$ & $0.42(0.1)^{a, B}$ & $1.35(0.4)^{b, B}$ & $1.33(0.2)^{b, B}$ \\
\hline
\end{tabular}

*Different letters, lower case letters on the line and capital letters in the column indicate statistically significant difference $(P<.05)$.

Table 4. Means (standard deviation) of microhardness (KHN) of aged samples (2-way ANOVA, Bonferroni, P<.05).

\begin{tabular}{lcccc}
\hline & Initial & Post-aging & Post-bleaching & 15 days after bleaching \\
\hline Charisma & $74.9(8.2)^{a, A}$ & $92.8(2.3)^{b, A}$ & $89.2(6.0)^{a b, A}$ & $86.2(3.4)^{a b, A}$ \\
Filltek Supreme 350 & $86.2(8.2)^{a, A}$ & $134.8(6.8)^{b, B}$ & $115.3(8.5)^{c, B}$ & $114.5(8.1)^{c, B}$ \\
Heliomolar & $43.0(6.1)^{a, B}$ & $61.2(10.7)^{b, C}$ & $107.0(23.1)^{c, B}$ & $93.6(15.4)^{c, A}$ \\
\hline
\end{tabular}

*Different letters, lower case letters on the line and capital letters in the column indicate statistically significant difference $(\mathrm{P}<.05)$. 
particle/resinous matrix bonding system may interfere in its properties. ${ }^{33}$ In that way, the significant color alteration of the Charisma composite can be related to the largest size and the largest concentration of the load particles in its composition. Smaller particles, with smaller load concentration in a composite, promote a larger light reflection among the particles. ${ }^{34}$ The size of the inorganic particles is also related to the color alteration, once composites formulated with large particles are more susceptible to water sorption and color alteration. Several studies have demonstrated that the composites allow solvents to penetrate into the resinous matrix or in the interface matrix/particles. When hydrolytic degradation of the polymeric network occurs, this interface can be altered and, consequently, to modify the light dispersal. ${ }^{20,35}$

According to Ferracane, ${ }^{35}$ as greater the volume of particles in the composite formulation, lower the degree of conversion presented. Consequently, the polymer formed would have a larger quantity of remaining double-bonds and lower quantity of formed bonds. Therefore, this composite will be more predisposed to the action of the solvent (water), as there will be a greater free volume for water action, which will penetrate into the resinous matrix, causing "swelling" or relaxation of these bonds, in an effect known as plasticization. The solvent inside may cause resinous matrix and particle/matrix interface deterioration. ${ }^{35}$ Plasticization will promote, besides the decrease of the composite hardness, greater color alteration, due to the presence of water inside the resinous matrix after AAA procedure. It was what happened with the Charisma composite in this study, which presented greater color alteration and smaller microhardness after bleaching, result that remained after 15 days.

In the present study, the clinical acceptance value of $\Delta E<3.3$ for esthetic restorative materials was used; a value adopted by other authors, ${ }^{36}$ and one exceeded by all the materials, with the exception of Filtek Supreme.

The other hypothesis tested in the study was that the use of the bleaching agent would not alter the surface roughness or microhardness of the composites. With regards to surface roughness, bleaching caused an increase in roughness, with statistically significant results in comparison with the initial situation $(P<.05)$. Nevertheless, the changes were small and material dependent, and the results are in agreement with other studies. 5,16 Bailey \& Swift ${ }^{9}$ suggested that the increase in roughness could be as a result of loss of resinous matrix rather than load particles.

The bleaching agent used in the study was carbamide peroxide-based, which breaks down into urea and hydrogen peroxide. This in turn forms free radicals that can eventually form water and accelerate the hydrolytic degradation of the composite, resulting ${ }^{35,37}$ in bonding failure between the resinous matrix and load particles, increasing the surface roughness of the composite. ${ }^{16}$

Zanin et a ${ }^{38}$ verified the color stability and surface roughness of indirect composite resins submitted to AAA for 384 hours. The authors verified that all the studied composites presented both color alteration and increase in surface roughness, and concluded that these properties are closely related. However, in the present study, it could be observed that the composites evaluated did not present statistically significant increase $(P>.05)$ in the surface roughness values after AAA. The relationship between surface roughness and color stability described by Zanin et al, ${ }^{38}$ did not occur in this case, since the composites Charisma and Heliomolar presented high $\Delta \mathrm{E}$ values, ${ }^{21}$ but low levels of surface roughness.

Based on these findings, it could be concluded that there was no direct relationship between surface roughness and color stability of the composites. Both AAA and the bleaching agent were capable of producing rougher surfaces, which would cause a more significant color alteration. It could be observed that the color alteration that occurred in all of the composites could be more related to the intrinsic alterations that occurred within the specimens, rather than on their surfaces. ${ }^{11}$

With regards to microhardness, there was a significant increase $(P<.05)$ after AAA, result of a process of post-polymerization caused by the action of temperature $\left(50^{\circ} \mathrm{C}\right)$ and water condensation. ${ }^{1,39,40}$ After bleaching, there was a significant reduction $(P<.05)$ in microhardness for Filtek Supreme, which was expected, since this composite presents a high concentration of resinous matrix to be oxidized by the hydrogen peroxide. $9,10 \mathrm{Howev}$ er, there are discrepancies between these results and those of other studies, due to the diversity of 
studied materials; since some are more susceptible to alterations than others. ${ }^{41,42}$ Moreover, the effect of bleaching may be different, as one of the limitations of the study was that the bleaching agent was not diluted in saliva, which could have diminished its action. Therefore, the second hypothesis of the study could not be accepted, as the bleaching agent produced surface alterations in the studied composites.

\section{CONCLUSIONS}

From the results found and considering the limitations of this study, it was concluded that:

- AAA promoted color alterations considered clinically unacceptable for Charisma and Heliomolar and bleaching promoted significant color alteration only for Charisma.

- The home bleaching agent produced an increase in the surface roughness values of the studied composites, an alteration that remained 15 days after bleaching.

- There was an increase in the microhardness values of the composites after AAA. After bleaching there was a significant reduction $(P<.05)$ for the nanoparticle composite. Fifteen days after bleaching, there was no significant alteration in microhardness ( $P>$.05).

- Composite alterations promoted by the bleaching agent are material dependent.

\section{REFERENCES}

1. Okte Z, Villalta P, Garcia-Godoy F, Lu H, Powers JM. Surface hardness of resin composites after staining and bleaching. Oper Dent 2006;31:623-628.

2. Berga-Caballero A, Forner-Navarro L, Amengual-Lorenzo J. At-home vital bleaching: a comparison of hydrogen peroxide and carbamide peroxide treatments. Med Oral Patol Oral Cir Bucal 2006;11:E94-E99.

3. Denehy G E, Swift E J Jr. Single-tooth home bleaching. Quintessence Int 1992;23:595-598.

4. Haywood VB. History, safety, and effectiveness of current bleaching techniques and applications of the nightguard vital bleaching technique. Quintessence Int 1992;23:471-488.

5. Polydorou 0, Hellwig E, Auschill TM. The effect of different bleaching agents on the surface texture of restorative materials. Oper Dent 2006;31:473-480.
6. Lynch E, Sheerin A, Samarawickrama DY, Atherton MA, Claxson AW, Hawkes J, Haycock P, Naughton D, Seymour KG, Burke FM. Molecular mechanisms of the bleaching actions associated with commercially-available whitening oral health care products. J Ir Dent Assoc 1995;41:94-102.

7. Villalta P, Lu H, Okte Z, Garcia-Godoy F, Powers JM. Effects of staining and bleaching on color change of dental composite resins. J Prosthet Dent 2006;95:137-142.

8. Mujdeci A, Gokay 0. Dental effects of home bleaching gels and whitening strips on the surface hardness of resin composites. Am J Dent 2005;18:323-326.

9. Bailey SJ, Swift EJ Jr. Effects of home bleaching products on composite resins. Quintessence Int 1992;23:489-494.

10. Cullen DR, Nelson JA, Sandrik JL. Peroxide bleaches: effect on tensile strength of composite resins. J Prosthet Dent 1993;69:247-249.

11. Garcia-Godoy F, Garcia-Godoy A, Garcia-Godoy F. Effect of bleaching gels on the surface roughness, hardness, and micromorphology of composites. Gen Dent 2002;50:247250.

12. Yap AU, Wattanapayungkul P. Effects of in-office tooth whiteners on hardness of tooth-colored restoratives. Oper Dent 2002;27:137-141.

13. Basting RT, Fernandez YFC, Ambrosano GM, de Campos IT. Effects of a $10 \%$ carbamide peroxide bleaching agent on roughness and microhardness of packable composite resins. J Esthet Restor Dent 2005;17:256-262.

14. Polydorou O, Monting JS, Hellwig E, Auschill TM. Effect of in-office tooth bleaching on the microhardness of six dental esthetic restorative materials. Dent Mater 2007;23:153158.

15. Turker SB, Biskin T. Effect of three bleaching agents on the surface properties of three different esthetic restorative materials. J Prosthet Dent 2003;89:466-473.

16. Wattanapayungkul P, Yap AU, Chooi KW, Lee MFLA, Selamat RS, Zhou RD. The effect of home bleaching agents on the surface roughness of tooth-colored restoratives with time. Oper Dent 2004;29:398-403.

17. Pires-de-Souza FCP, Garcia LFR, Hamida HM, Casemiro LA. Color stability of composites subjected to accelerated aging after curing using either a halogen or a light emitting diode source. Braz Dent J 2007;18:119-123.

18. ASTM STANDARDS G154-00A. Standard practice for operating fluorescent light apparatus for UV exposure of nonmetallic materials. Annual Book of ASTM Standards, v 14 ed (04). Philadelphia, PA, 2006, p. 646-654.

19. Douglas RD. Color stability of new-generation indirect resins for prosthodontic application. J Prosthet Dent 2000;83:166-170. 
20. Vichi A, Ferrari M, Davidson CL. Color and opacity variations in three different resin-based composite products after water aging. Dent Mater 2004;20:530-534.

21. Ruyter IE, Nilner K, Moller B. Color stability of dental composite resin materials for crown and bridge veneers. Dent Mater 1987;3:246-251.

22. Hayashi H, Maejima K, Kezuka K, Ogushi K, Kono A, Fusayama $T$. In vitro study of discoloration of composite resins. J Prosthet Dent 1974;32:66-69.

23. Leinfelder KF, Sluder TB, Stockwell CL, Strickland WD, Wall JT. Clinical evaluation of composite resins as anterior and posterior restorative material. J Prosthet Dent 1975;33:407-416.

24. Ameye C, Lambrechts $P$, Vanherle G. Conventional and microfilled composite resins. Part I: color stability and marginal adaptation. J Prosthet Dent 1981;46:623-630.

25. Hachiya Y, Iwaku M, Hosoda H, Fusayama T. Relation of finish to discoloration of composite resins. J Prosthet Dent 1984;52:811-814

26. Powers JM, Bakurat MM, Ogura H. Color and optical properties of posterior composites under accelerated aging. Dent Mater J 1985;4:62-67.

27. Powers JM, Bakus ER, Goldberg AJ. In vitro color change of posterior composites. Dent Mater 1988;4:151-154.

28. Dietschi D, Campanile G, Holz J, Meyer JM. Comparison of the color stability of ten new-generation composites: an in vitro study. Dent Mater 1994;10:353-362.

29. Miller LL. Shade matching. J Esthet Dent 1993;5:143-153.

30. Miller LL. Esthetic dentistry development program. J Esthet Restor Dent 1994;6:47-60.

31. Rosentritt M, Lang R, Plein T, Behr M, Handel G. Discoloration of restorative materials after bleaching application. Quintessence Int 2005;36:33-39.

32. Abu-Bakr N, Han L, Okamoto A, Iwaku M. Color stability of compomer after immersion in various media. $J$ Esthet Dent 2000;12:258-263

33. Ikeda T, Nakanishi A, Yamamoto T, Sano H. Color differences and color changes in Vita Shade tooth-colored restorative materials. Am J Dent 2003;16:381-384.

34. Schulze KA, Marshall SJ, Gansky SA, Marshall GW. Color stability and hardness in dental composites after accelerated aging. Dent Mater 2003;19:612-619.

35. Ferracane JL. Hygroscopic and hydrolytic effects in dental polymer networks. Dent Mater 2006;22:211-222.

36. Lee YK, Powers JM. Color changes of resin composites in the reflectance and transmittance modes. Dent Mater 2007;23:259-264.

37. Soderholm KJ, Zigan M, Ragan M, Fischlschweiger W, Bergman M. Hydrolytic degradation of dental composites. J Dent Res 1984;63:1248-1254.
38. Zanin FR, Garcia LFR, Casemiro LA, Pires-de-Souza FCP. Effect of artificial accelerated aging on color stability and surface roughness of indirect composites. Eur J Prosthodont Restor Dent 2008;16:10-14.

39. Braden M, Pearson GJ. Analysis of aqueous extract from filled resins. J Dent 1981;9:141-143.

40. Martin N, Jedynakiewicz NM, Fisher AC. Hygroscopic expansion and solubility of composite restoratives. Dent $\mathrm{Ma}$ ter 2003;19:77-86.

41. Swift EJ Jr, Perdigao J. Effects of bleaching on teeth and restorations. Compend Contin Educ Dent 1998;19:815-820.

42. Yu B, Lee YK. Translucency of varied brand and shade of resin composites. Am J Dent 2008;21:229-232. 